\title{
Uber eine interessante Froststruktur im episodisch-solifluidal bewegten Boden während der Würmeiszeit
}

\author{
Von J.-H. Henke, Bad Oeynhausen
}

Mit 1 Abbildung im Text

Zusammenfassung: Im östlichen Teil der Stadt Bielefeld wurde ein Frostkessel festgestellt, der ca. $3 \mathrm{~m}$ in rote und grüne Keupermergel hineinreichte, die nur episodisch während der Würmeiszeit bewegt wurden.

Seine Füllmasse bestand neben glazialem Geröll aus gelben Sanden und 4 Schichten brauner Sande mit tonigen Bestandteilen (2,5, cm mächtig), die sich warwenartig in gleichen Abständen in die gelben Sande einschalteten.

Die braunen Schichten unterlagen einer Frosthebung von 5,5 cm in der Mitte, die als Auswirkung der hydratisierenden Teilchen des Mineralbodens während eines Kaltstadials in der Würmeiszeit erklärt wird.

Während die gelben Sande durch Schmelzwässer bei sommerlichem Auftauen in den Spalt eingeschwemmt wurden, sind die tonigen Bestandteile vermutlich als verwitterte Keupermergel nach Austrocknen des Bodens eingeweht.

$\mathrm{Da}$ Lößlehm über einer Steinsohle liegt, die keinerlei frostdynamischen Vorgängen mehr unterworfen war, wird angenommen, daß sich der Frostkessel in der zweiten Phase der Würmeiszeit, dem Hochglazial, gebildet hat.

$\mathrm{Sum}$ m a ry : A frost-kettle is described as situated in the eastern part of the town of Bielefeld and reaching about $3 \mathrm{~m}$ into green and red marl, which had been moved episodically during the Würm glaciation.

The frost-kettle was filled with yellow sand and glacial rubble as well as 4 layers' of brown sand with small particles of clay $(2.5 \mathrm{~cm}$ thick), inserted at regular intervals into the yellow sand.

The brown layers showed a frost-upheaval of $5.5 \mathrm{~cm}$ in the centre due to hydration in a cold stadium of the last glaciation.

While the yellow sand was washed into the cleft during the summer thawing periods, the clay particles of the brown layers consisting of weathered marl were blown into it after the soil had been dried.

As loess-loam lies above a stone-layer showing no frost-dynamic modifications, the frost-kettle must have been formed during the second phase of the Würm glaciation, i. e. the high-glacial period.

Auf der Nordseite des Teutoburger Waldes wurden im Spätsommer 1963 auf der Kreuzbrede im Osten der Stadt Bielefeld bei Ausschachtungsarbeiten für einen Neubau verschiedene Froststrukturen, wie Eiskeile, Frostkessel und Eislinsen beobachtet.

Die Erscheiungsformen reichten bis zu einer durchschnittlichen Tiefe von $4 \mathrm{~m}$ in rote und grüne Keupermergel, die durch solifluidale Bewegungen während der Würmeiszeit vermengt wurden, wie aus einer die Mergel überlagernden Steinsohle und dem Lößlehm geschlossen werden konnte. Die Hangneigung beträgt hier $2^{\circ}$.

Im folgenden soll nun ein Frostkessel näher beschrieben werden, der an der Nordwand der Baugrube aufgeschlossen war. Er war der Länge nach durchschnitten, bog dann aber in 1,25 m Tiefe seitlich in die Keuperwand aus, wo er noch ca. $1 \mathrm{~m}$ weiter verfolgt werden konnte. Die Breite des Frostkessels betrug ca. $40 \mathrm{~cm}$; zur Tiefe hin stellte sich eine allmähliche Verschmälerung ein. Interessant war die Zusammensetzung und Struktur der Füllmasse. Im untersten Teil des Frostkessels befanden sich zunächst gelbe Sande mit saaleeiszeitlichen Geröllen, die sich zu über 50\% aus blauen Graniten und Hälleflinten zusammensetzten (Durchmesser bis $4 \mathrm{~cm}$ ). Alle Gerölle waren kantengerundet, was für periglaziales Klima typisch ist. Von $55 \mathrm{~cm}$ Tiefe ab (von der Oberfläche aus gemessen), schalteten sich vier 2,5 cm mächtige braune Sandschichten in Abständen von $20 \mathrm{~cm}$ warwenartig in die gelben Sande ein. Die braunen Schichten waren ca. 5,5 cm in der Mitte ge- 
hoben und wiesen an den oberen Rändern eine leichte Häufung saaleeiszeitlichen Materials auf, das offenbar durch die Hebung seitlich versetzt wurde. Die gelben und die braunen Sande waren zu ca. 90\% mittel- bis feinkörnig. Auf die gröberen Sande $(>0,5 \mathrm{~mm})$ entfielen nur ca. 5\%. Den Rest bildeten bei den gelben Sanden feinste Korngrößen $(<0,1 \mathrm{~mm})$, bei den braunen Sanden tonige Bestandteile. Die Analyse ergab, daß beide Sandschichten zu über $90 \%$ aus Siliziumdioxyd bestanden. Außerdem wurden Feldspat und Spuren von Eisen nachgewiesen. Beide Sande waren kalkfrei.

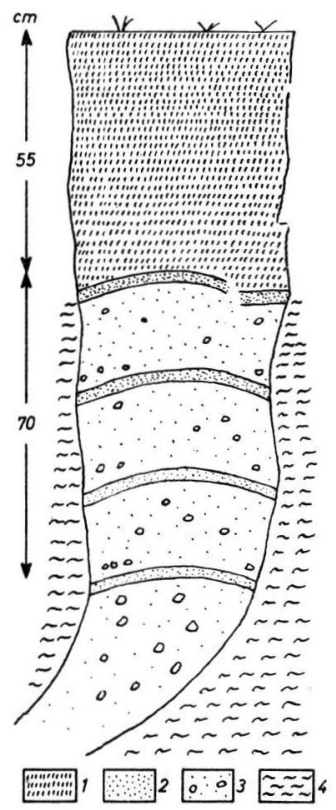

Abb. 1. Querschnitt durch einen Frostkessel in Bielefeld.

$1=$ Lößlehm; $2=$ brauner Sand mit tonigen Bestandteilen; $3=$ gelber Sand und glaziales Geröll; 4 = stark verknetete rote und grüne Keupermergel.

Die braunen Sandschichten hatten durch Eisenoxydhydrat eine Verfestigung erfahren, so daß sie zur Zeit der Untersuchung, bis auf die abschließende Schicht, die eine leichte Deformierung an der rechten Seite aufwies, vollkommen intakt waren. Diese Deformierung muß als eine Folge des Gewichtes der auflastenden Lößschichten bei mäßigem Auftauen im Spätglazial verstanden werden. Die Frosthebung dieser Schichten wird im Sinne von E. Schenk (1955, S, 175) als Auswirkung der hydratisierenden Teilchen des Mineralbodens, d. h. der tonigen bzw. kolloidalen Teilchen erklärt. Die Keupermergel dürften genügend Wassermengen für die Eisbildung zur Verfügung gestellt haben. Da der Lößlehm über einer Steinsohle liegt, die keinerlei frostdynamischen Vorgängen mehr unterworfen war, wird sich der Frostkessel in der 2. Phase der Würmeiszeit, dem Hochglazial, gebildet haben (vgl. auch H. Poser, 1951, S. 32 ff.).

Die über dem Frostkessel fehlende Steinsohle beweist, daß der gelbe Sand mit dem nordischen Geröll bei sommerlichem oberflächlichen Auftauen durch Schmelzwässer eingeschlämmt worden ist. Die tonigen Bestandteile der braunen Sandschichten sind vermutlich nach Austrocknen des Bodens in den Spalt eingeweht. Bei dem Ton handelt es sich offenbar um verwitterte Keupermergel. E. Ackermann (1954, S. 130 ff.) berichtet über einen unter ähnlichen Umständen durch Wind umgelagerten Rotsand, den er als Verwitterungsprodukt des Buntsandsteins deutet. Einwehung und Einwaschung müssen in unserem Fall rhythmisch erfolgt sein. Über die Dauer des Vorganges kann jedoch keine verbindliche Aussage gemacht werden.

Aus der Tatsache, daß die Frosthebung im Frostkessel erhalten geblieben ist, wird man schließen müssen, daß sich die solifluidalen Vorgänge nach seiner Bildung nicht mehr 
wiederholt haben. Als weitere Anzeichen dafür, daß sich der Boden nicht kontinuierlich, sondern nur gelegentlich-episodisch bewegt hat, möchte ich wie J. BüDEL (1959, S. 310) die geringe Hangneigung von $2^{\circ}$, die großen Auftautiefen bis zu $4 \mathrm{~m}$, die intensive Vermengung und Verknetung der Mergel im oberen Teil der Gleitzone und die Bildung von Aufpressungen und Scherflächen im tieferen Bereich anführen. Die Entstehung des Frostkessels kann man sich abschließend wie folgt vorstellen: Eiskeilbildung und Erweiterung zum Frostkessel in einem Kaltstadial, Auftauen des Eises und Einschlämmen bzw. Einwehen dər Füllmasse während eines Interstadials und Frosthebung in einem erneut einsetzenden Kaltstadial.

$$
\text { Benutzte Literatur: }
$$

E. Ackermann: Gliederung, Kinematik und paläoklimatische Bedeutung der würmeiszeitlichen Ablagerungen in Göttingen. - Mitt. geol. Staatsinstitut Hamburg 23, S. 126-141, 1954. J. BüDEL: Periodische und episodische Solifluktion im Rahmen der klimatischen Solifluktionstypen. Erdkunde 13, S. 297-314, 1959.

H. Poser: Die nördliche Lößgrenze in Mitteleuropa und das spätglaziale Klima. - Eiszeitalter und Gegenwart 1, S. 27-55, 1951.

E. Schenk: Die periglazialen Strukturbildungen als Folgen der Hydrationsvorgänge im Boden. Eiszeitalter und Gegenwart 6, S. 170-184, 1955.

Manuskr. eingeg. 1. 5., 1964.

Anschrift des Verf.: J.-H. Henke, 497 Bad Oeynhausen, Walderseestraße 14. 